\title{
Death Losses for Lactating Cows in Herds Enrolled in Dairy Herd Improvement Test Plans
}

\author{
R. H. Miller, ${ }^{1}$ M. T. Kuhn, H. D. Norman, and J. R. Wright \\ Animal Improvement Programs Laboratory, Agricultural Research Service, USDA, Beltsville, MD 20705-2350
}

\begin{abstract}
Factors that affect frequency of death of lactating cows were studied for cows with records that terminated from 1995 through 2005. Analyses included effects of herd, year, month, parity, and lactation stage at lactation termination as well as cow breed and milk yield. A national data set $(15,025,035$ lactations in 45,032 herds) was analyzed with PROC GLM. Overall death frequency was $3.1 \%$ per lactation $(5.7 \%$ per cow). Death frequency increased by $1.6 \%$ from 1995 to 2005 , with a sudden increase of $0.9 \%$ from 2003 to 2004, probably because of a USDA requirement in late 2003 for euthanizing downer cows. Death frequency was $16.5 \%$ greater for lactations that terminated at $\leq 45 \mathrm{~d}$ than for those that terminated at $\geq 251 \mathrm{~d}$. Death frequency increased with parity ( $2 \%$ greater for eighth parity and later than for first parity) and with lactation milk yield $(0.4 \% / 1,000 \mathrm{~kg}$ for Holsteins and Jerseys and $0.5 \% / 1,000 \mathrm{~kg}$ for other breeds). Deaths were most frequent in July and least frequent in November. Within-herd breed differences (Holstein, Jersey, and other breeds) were small. The heritability of likelihood of death estimated from a sample of 79,162 Holstein cows was $1.3 \%$. Death losses are increasing, perhaps partly because of increased milk yield and more intensive management regimens.
\end{abstract}

Key words: death loss, death frequency, lactation termination

\section{INTRODUCTION}

The dairy industry is concerned that the frequency of death among lactating cows is increasing. Thomsen and Houe (2006) reviewed 19 European, North American, Australian, and New Zealand studies of dairy cow mortality and reported that mortality ranged from 1 to $5 \%$. They indicated the possibility of increasing mortality over time but did not analyze time trends for the

Received December 12, 2007.

Accepted May 8, 2008.

${ }^{1}$ Corresponding author: millerrh@juno.com populations reviewed. Thomsen et al. (2004) reported that death rates for Danish dairy cattle had increased from $2 \%$ in 1990 to $3.5 \%$ in 1999 .

Milian-Suazo et al. (1988) reported a death rate of $1.2 \%(0.1 \%$ for first-parity cows and $1.1 \%$ for mature cows) for 34 New York Holstein herds from 1981 through 1985. Gardner et al. (1990) reported a mortality rate of $2 \%$ for cow-years evaluated by the National Animal Health Monitoring System from 1986 to 1987 in 43 California herds. For Holstein lactation records that were processed by the Mid-States Dairy Records Processing Center (Ames, IA) from 1980 to 1991, Dematawewa and Berger (1998) reported a mortality rate of $2 \%$ during 305 -d lactations. Karuppanan et al. (1997) studied management records from 1987 to 1992 of 9 large (19,482 cows) dry-lot herds in California and found that death rate ranged from 0.8 to $6.4 \%$ based on cow-years. Young (2002) reported an increase in death rate in the northwestern United States from 5\% in 1994 for Holsteins and Jerseys to 8\% in 2001 for Holsteins and around 7\% for Jerseys. For DHI Holstein herds during 1998, Smith et al. (2000) reported death rates of $7.7 \%$ for the South, $7.0 \%$ for the Mid-South, and $5.9 \%$ for the North. For 20 New York herds, Stone et al. (2006) reported an annual death rate of $8.1 \%$, with a range of 3.5 to $16.8 \%$ among herds.

Reasons for the increase in death rate are unclear. Thomsen and Houe (2006) found 10 studies that reported the cause of death as unknown for 16 to $46 \%$ of deaths, accidents for 5 to $13 \%$, udder and teat disorders for 8 to $25 \%$, and metabolic disorders for 8 to $18 \%$. Nørgaard et al. (1999) and Sigurdarson and Thorsteinsson (1990) attributed the increase in mortality rate to a greater level of physiological stress due to greater milk yield and concentrate consumption. However, Young (2002) found that death rates were greater for the lowest $25 \%$ of cows for milk yield compared with the top $25 \%$. In an investigation of factors that affected voluntary and involuntary culling in 186 Holstein Wisconsin herds that expanded extensively between 1994 and 1998, Weigel et al. (2003) reported an increase in relative risk of involuntary culling for high-producing cows compared with average cows from 0.50 for 1981 to 1989 to 0.68 for 1996 to 2000 . 
Table 1. Variance analyses for a sample of 1,645 herds $^{1}$

\begin{tabular}{lcc}
\hline Effect & GENMOD $x^{2}$ & GLM $F$-values \\
\hline Year & $1,772.2$ & 180.5 \\
Month & 295.5 & 25.3 \\
Parity & $2,574.3$ & 349.4 \\
Lactation stage & $72,595.3$ & $28,504.2$ \\
Breed & 6.4 & 6.3 \\
\hline
\end{tabular}

${ }^{1}$ All effects significant at $P<0.01$.

A regulatory change in the United States at the end of December 2003 required euthanasia of downer cows to prohibit use in human food supply channels (USDA, 2003). That change would be expected to shift DHI reporting of reason for lactation termination from "sold for slaughter or salvage" (formerly "sold for beef") to "died" (Animal Improvement Programs Laboratory, 2007), as noted by Fetrow et al. (2006). The objectives of this study were to assess the importance of the effects of year, season, parity, lactation stage, breed, and milk yield on death frequency among US lactating cows and to estimate the heritability of death rate.

\section{MATERIALS AND METHODS}

Expression of death rates varies but is most frequently given as number of deaths relative to total number of cows at risk during a specified time period. Fetrow et al. (2006) listed several alternatives for expressing culling incidence, such as frequency per cow-year, cow-month, or lactation. Thomsen and Houe (2006) found that number of dead cows per 100 cowyears and mortality risk (number of dead cows per 100 lactations) were the most common expressions used. They concluded that lactational mortality risk is the most desirable measure of mortality. For this study, death frequency was expressed as the probability of a lactation ending in death [i.e., incidence was binomial $(0=$ live, $1=$ died during lactation $)$ based on the presence or absence of a termination code of 6 (cow died on the dairy, including downer cows that were killed) in DHI lactation data that are provided to USDA (Animal Improvement Programs Laboratory, 2007)]. The definition of termination codes has changed over time, and only recently have cows that died before first test or after 305 DIM been reported. The omission of deaths before first test and during dry period causes death frequency to be underestimated.

A sample of herds was chosen for a preliminary variance analysis based on the units position of the herd code. Records for lactations that terminated during 1995 through 2005 were extracted for those herds from the USDA national database maintained by the Animal Improvement Programs Laboratory (Beltsville,
Table 2. Observed death frequencies by year, month, and lactation stage at termination of the lactation record and by parity

\begin{tabular}{|c|c|}
\hline Category & Death frequency (\%) \\
\hline \multicolumn{2}{|l|}{ Year } \\
\hline 1995 & 1.96 \\
\hline 1996 & 2.18 \\
\hline 1997 & 2.60 \\
\hline 1998 & 2.92 \\
\hline 1999 & 2.92 \\
\hline 2000 & 2.97 \\
\hline 2001 & 3.68 \\
\hline 2002 & 3.83 \\
\hline 2003 & 3.54 \\
\hline 2004 & 4.30 \\
\hline 2005 & 4.63 \\
\hline \multicolumn{2}{|l|}{ Month } \\
\hline January & 3.13 \\
\hline February & 3.45 \\
\hline March & 3.35 \\
\hline April & 3.10 \\
\hline May & 2.98 \\
\hline June & 2.98 \\
\hline July & 3.30 \\
\hline August & 3.53 \\
\hline September & 3.18 \\
\hline October & 2.84 \\
\hline November & 2.80 \\
\hline December & 3.09 \\
\hline \multicolumn{2}{|l|}{ Lactation stage } \\
\hline$\leq 45 \mathrm{DIM}$ & 17.3 \\
\hline 46 to 90 DIM & 10.1 \\
\hline 91 to $150 \mathrm{DIM}$ & 8.5 \\
\hline 151 to 250 DIM & 6.5 \\
\hline$\geq 251 \mathrm{DIM}$ & 0.9 \\
\hline \multicolumn{2}{|l|}{ Parity } \\
\hline 1 & 2.05 \\
\hline 2 & 2.66 \\
\hline 3 & 3.72 \\
\hline 4 & 4.38 \\
\hline 5 & 4.83 \\
\hline 6 & 5.78 \\
\hline 7 & 5.92 \\
\hline$\geq 8$ & 6.40 \\
\hline
\end{tabular}

$\mathrm{MD})$. Records for lactations that ended $<1 \mathrm{yr}$ before the herd stopped participating in DHI testing or for lactations without estimates of 305-d, mature-equivalent milk yield were excluded, which resulted in eliminating any recent reports of death before first test. Two analysis procedures were compared using the sample set of herds: PROC GENMOD and PROC GLM (SAS Institute Inc., 2004). Although PROC GENMOD accommodates binomially distributed variables, it requires a great deal more computer resources for an iterative solution. To avoid failure of GENMOD to converge, herds with $<400$ total lactations across years or a death rate of $<0.3 \%$ were excluded. Records from 2,131,035 lactations of 971,763 cows in 1,645 herds remained for analysis.

The model included effects at lactation termination for herd, year, month, parity $(1,2, \ldots 7, \geq 8)$, and lactation stage $(\leq 45,46$ to 90,91 to 150,151 to 250 , and 


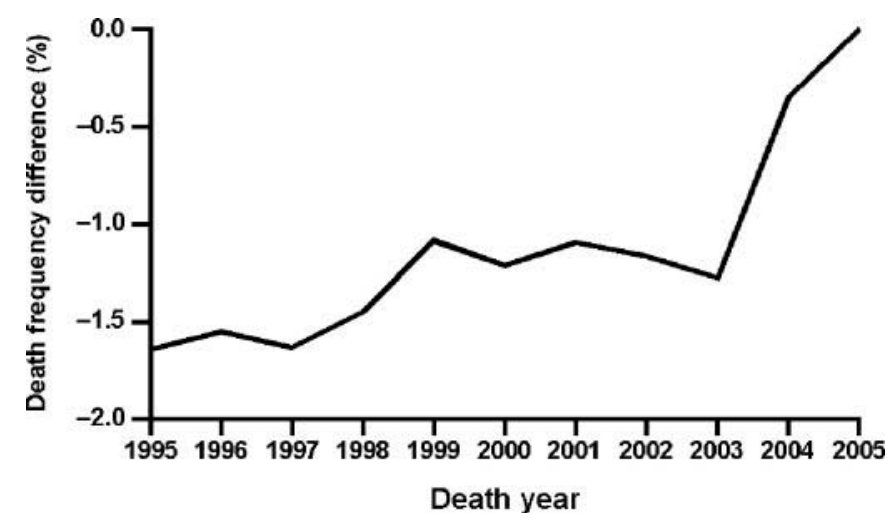

Figure 1. Least squares estimates of across-breed difference in frequency of death during lactation relative to 2005 by death year.

$\geq 251$ DIM) as well as an effect for cow breed (Holstein, Jersey, and other). A second model added regressions on milk yield within breed. After analysis of the sample data set, the model was applied to the national data set for 1995 through 2005 (45,032 herds with 15,025,035 lactations, of which 472,039 were terminated by death). An additional analysis of means of herd-years with $\geq 50$ lactations included effects of region (Mideast, Midwest, Mountain-Prairie, Northeast, Northwest, Southeast, and Southwest) as defined by Hare et al. (2004), state, and herd size (50 to 99,100 to $199, \ldots, 900$ to 999 , $\geq 1,000$ lactations per year).

Heritability was estimated by REML with the program AIREMLF90. Data were a sample of 79,162 Holstein cows from 135 randomly selected herds. The model was similar to that described above but excluding effects of breed and milk yield and including a permanent cow environmental effect.

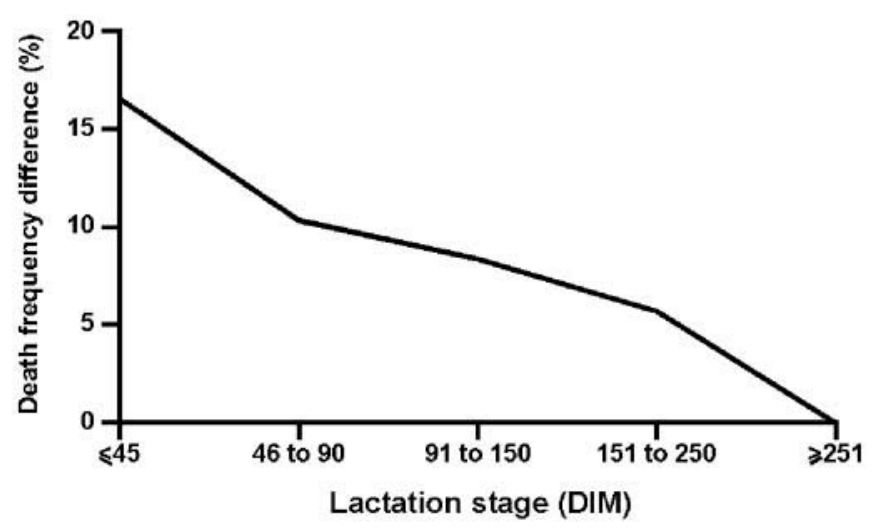

Figure 2. Least squares estimates of across-breed difference in frequency of death during lactation relative to $\geq 251$ DIM by lactation stage.

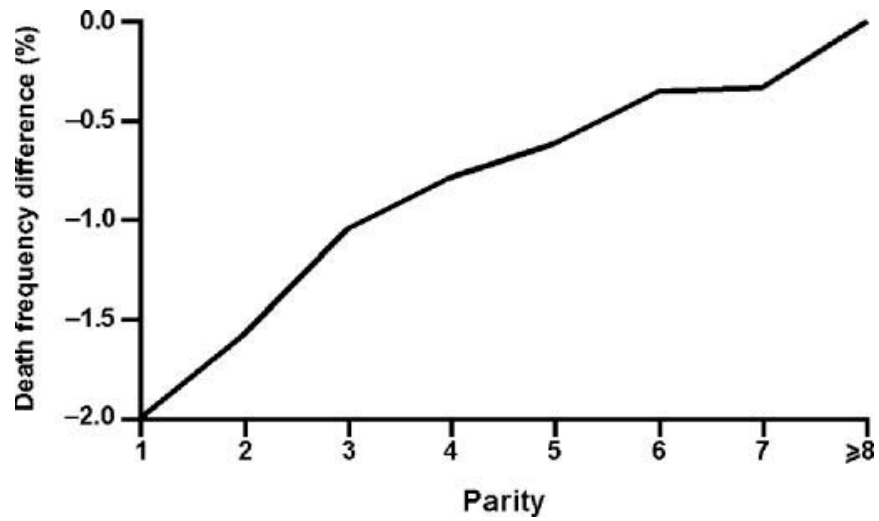

Figure 3. Least squares estimates of across-breed difference in frequency of death during lactation relative to $\geq 8$ parities by parity.

\section{RESULTS AND DISCUSSION}

Results of GENMOD and GLM variance analysis of the sample data set are in Table 1. Both analysis procedures ranked the model effects similarly: lactation stage, parity, year, month, and breed (largest to smallest). All effects were significant $(P \leq 0.01)$, but lactation stage was the largest effect by far, especially for the GLM analysis; breed differences were very small. The total coefficient of determination for the model was $8.4 \%$, with $5.6 \%$ of variation explained by lactation stage and $<0.1 \%$ explained by each of the other effects in the model. Because results were similar and because GENMOD is computationally more demanding, GLM was used to analyze the large national data set.

For the national data set, observed mean death frequency was $3.1 \%$ on a lactation basis and $5.7 \%$ on a cow basis. Observed death frequencies by year, month, and lactation stage at record termination and by parity are in Table 2.

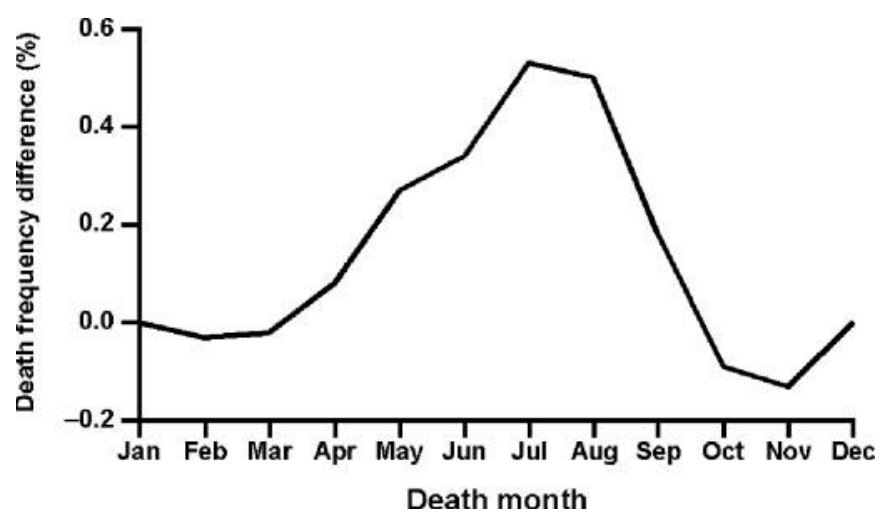

Figure 4. Least squares estimates of across-breed difference in frequency of death during lactation relative to December by death month. 


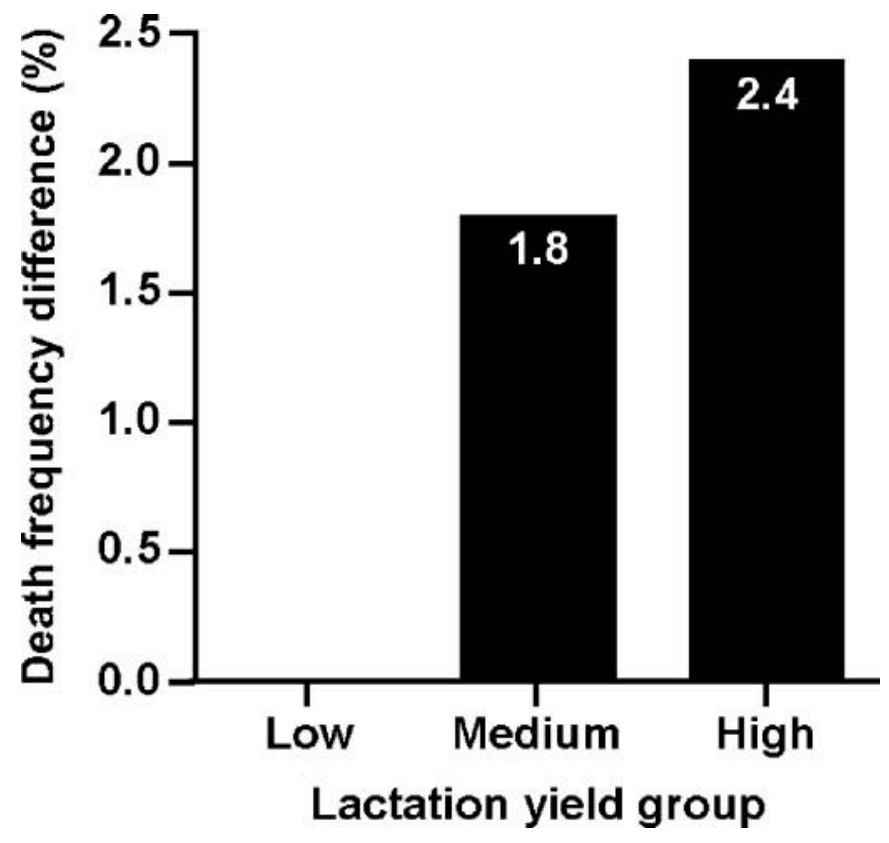

Figure 5. Least squares estimates of Holstein difference in frequency of death during lactation relative to low lactation milk yield by lactation yield group (low $=\leq 6,349 \mathrm{~kg}$; medium $=6,350$ to 13,608 $\mathrm{kg}$; high $=\geq 13,609 \mathrm{~kg}$ ).

Effect of year on death frequency relative to 2005 is shown in Figure 1 for the national data set. Year effect was greatest for 2005 and least for 1995 . The increase of $1.64 \%$ from 1995 to 2005 agrees with the $1.5 \%$ increase reported by Thomsen et al. (2004) for Danish cows that died during the 1990s. The estimated trend mirrors observed losses (Table 2): 1.96\% in 1995 and $4.63 \%$ in 2005 . The increase in effect of year on death frequency was rather consistent over time except for the large increase from 2003 to $2004(0.92 \%)$. That increase likely resulted from the USDA requirement that nonambulatory cows be killed (USDA, 2003) and a shift from cows reported as "sold for beef" to "died." Although frequency of downer cows killed before 2004 is unknown, the $0.9 \%$ increase indicates a substantial new economic loss for dairy producers.

The shortest lactations were associated with the greatest death frequency (Figure 2), which reflected that only cows that died or were culled had short lactations in the national data set. Compared with lactations that were terminated at $\geq 251$ DIM, death frequency was $16.5 \%$ greater for lactations that terminated at $\leq 45$ DIM. Because the data did not include deaths before first test, deaths at $\leq 45$ DIM were underestimated. However, lactations of $\leq 45$ DIM would be terminated only by death or voluntary disposal; thus, the high death frequency for that lactation stage is a comparison with frequency of voluntary disposal. The death frequency for lactations that were terminated between 46 and 90 DIM was greater (10.3\%) than for lactations that terminated at $\geq 251$ DIM. Relative to total deaths, $22.7 \%$ of deaths occurred at $\leq 45$ DIM, $21.9 \%$ between 46 and 90 DIM, $17.1 \%$ between 91 and 150 DIM, $18.0 \%$ between 151 and 250 DIM, and $20.3 \%$ at $\geq 251$ DIM. Mean DIM at death was $148.5 \mathrm{~d}$. When those percentages are expressed as death frequency per day of lactation, they suggest that the likelihood of death decreases after 90 DIM. Greater death frequency in early lactation was reported by Milian-Suazo et al. (1988), Menzies et al. (1995), Stevenson and Lean (1998), Thomsen et al. (2004), and Stone et al. (2006).

As anticipated, death frequency steadily increased from first to subsequent parities (Figure 3). Death frequency for first parity was $1.99 \%$ lower than for eighth parity or later. Thomsen et al. (2004) reported that mortality risk for older cows (third parity and later) was approximately twice that for younger cows. Stevenson and Lean (1998) also reported greater mortality among older cows. The unreported deaths before first test may be more frequent for first-parity calvings.

Differences among calendar months in which death occurred (Figure 4 ) were small but significant $(P \leq 0.01)$. Deaths were least frequent in November and most frequent in July ( $0.66 \%$ greater than for November). The trend was for a peak in death frequency during July and August, a decline through November, and then a rise through winter and spring. Two seasons appear to be May through September and October through April. Although estimated differences for model effects generally closely paralleled observed frequencies (Table 2), month trend was an exception. Observed frequencies were greatest for records that ended in August (3.53\%) and February (3.45\%). However, the observed frequencies were subject to bias from herd effects; therefore, no clear interpretation is possible. Little previous information is available for seasonal differences in mortality. Stone et al. (2006) reported deaths due to severe injuries incurred by cows that fell on icy surfaces during winter for 20 New York herds. Seasonal differences may have variable patterns among geographical regions of the United States.

Within-herd breed differences were small. Death frequency for breeds other than Holstein and Jersey was slightly smaller $(0.22 \%)$ than for Holsteins but slightly larger $(0.17 \%)$ than for Jerseys. Regression of death frequency on lactation milk yield was $0.37 \% / 1,000 \mathrm{~kg}$ for Holsteins, $0.41 \% / 1,000 \mathrm{~kg}$ for Jerseys, and 0.47\%/1,000 $\mathrm{kg}$ for other breeds ( $t$-values of $>30$ ). The positive regression supports the premise that increased milk yield per cow contributes to the historical increase in death rate. Inclusion of milk yield in the model reduced the magnitude of breed differences in death frequency. 


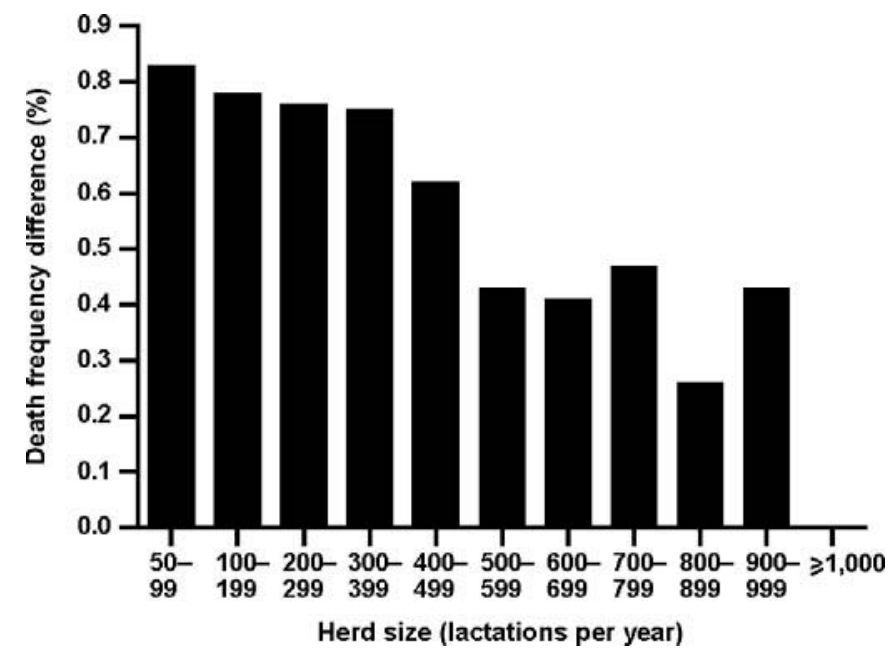

Figure 6. Least squares estimates of across-breed difference in frequency of death during lactation relative to herd size of $\geq 1,000$ lactations per year by herd size.

To illustrate change in death frequency with increasing milk yield, lactation milk yield was categorized as low ( $\leq 6,349 \mathrm{~kg})$, medium (6,350 to $13,608 \mathrm{~kg})$, and high $(\geq 13,609 \mathrm{~kg})$. Results for Holsteins (Figure 5) show that the relationship between milk yield and death frequency is not linear. Death frequency was $1.8 \%$ greater for medium-yield cows than for low-yield cows, whereas death frequency for high-yield cows was only $0.6 \%$ greater than for medium-yield cows.

Least squares estimates for death frequency by herd differed by $24.1 \%$ between herds with the lowest and greatest death rates. For states with $\geq 10$ herds in the sample, means of herd solutions were greatest for South Dakota (1.5\%) and Oklahoma (1.4\%). Estimated death frequency was greatest for the Southeast and lowest for the Northeast (difference of 5\%). Analysis of herdyear means disclosed a small but consistent decrease in death frequency as herd size increased (Figure 6). Herd-years with $\geq 1,000$ lactations per year had a death frequency that was $0.8 \%$ lower than herd-years with 50 to 99 lactations per year.

Six iterations were required for heritability estimation. The estimate was $1.3 \%$; thus, only about $1 \%$ of the variation in the likelihood that a cow will die during lactation is genetic. When converted to the underlying scale (Dempster and Lerner, 1950), the heritability estimate was $8 \%$. If death loss was expressed on a lifetime rather than lactation basis, genetic variation might be proportionately greater. Death frequency contributes to the trait of productive life (VanRaden et al., 2006) and is subject to some indirect selection pressure. Nevertheless, management factors must be considered the major contributors to death frequency.

\section{CONCLUSIONS}

Frequency of deaths per lactation increased by $1.6 \%$ from 1995 to 2005 . A sudden increase of $0.9 \%$ from 2003 to 2004 most likely was caused by USDA regulations in late December 2003 that required euthanizing downer cows because of concerns about bovine spongiform encephalopathy. Death was more likely to occur during early lactation than later, even without data for deaths before first DHI test. Frequency of death increased with age ( $2 \%$ greater for eighth parity and later compared with first parity). Seasonal differences in death frequency were small, but more deaths occurred in July compared with December. Breed differences were negligible. High-producing cows were more likely to die than low producers. Increased herd size was accompanied by lower death frequencies. Heritability of the likelihood of death during lactation was $1.3 \%$. Reducing death rate is primarily a problem of management; because the national data set lacked cause of death, specific management practices to decrease death losses could not be identified. Despite that low heritability, attempts to reduce death rate through genetic means likely would be of value because of the great economic loss from each incident.

\section{ACKNOWLEDGMENTS}

The cooperation of AgriTech Analytics (Visalia, CA), AgSource Cooperative Services (Verona, WI), Dairy Records Management Systems (Raleigh, NC), DHI Computing Services (Provo, UT), and Texas DHIA (College Station, TX) in supplying data through the National Genetic Improvement Program was invaluable. The AIREMLF90 program was made available by S. Tsuruta and I. Misztal, University of Georgia (Athens). The suggestions of 2 anonymous reviewers and A. J. Young, Utah State University (Logan), and the assistance of J. L. Hutchison and S. M. Hubbard, Animal Improvement Programs Laboratory (Beltsville, $\mathrm{MD})$, in manuscript review and preparation are appreciated.

\section{REFERENCES}

Animal Improvement Programs Laboratory. 2007. Reference 10. Cow destination group. http://aipl.arsusda.gov/formats/ref.10. html Accessed Nov. 15, 2007.

Dematawewa, C. M. B., and P. J. Berger. 1998. Genetic and phenotypic parameters for 305-day yield, fertility, and survival in Holsteins. J. Dairy Sci. 81:2700-2709.

Dempster, E. R., and I. M. Lerner. 1950. Heritability of threshold characters. Genetics 35:212-235.

Fetrow, J., K. V. Nordlund, and H. D. Norman. 2006. Invited review: Culling: nomenclature, definitions, and recommendations. J. Dairy Sci. 89:1896-1905. 
Gardner, I. A., D. W. Hird, W. W. Utterback, C. Danaye-Elmi, B. R. Heron, K. H. Christiansen, and W. M. Sischo. 1990. Mortality, morbidity, case-fatality, and culling rates for California dairy cattle as evaluated by the National Animal Health Monitoring System, 1986-87. Prev. Vet. Med. 8:157-170.

Hare, E., H. D. Norman, and J. R. Wright. 2004. Duration of herd participation in Dairy Herd Improvement milk recording in the United States. J. Dairy Sci. 87:2743-2747.

Karuppanan, P., M. C. Thurmond, and I. A. Gardner. 1997. Survivorship approaches to measuring and comparing cull rates for dairies. Prev. Vet. Med. 30:171-179.

Menzies, F. D., D. G. Bryson, T. McCallion, and D. I. Matthews. 1995. A study of mortality among suckler and dairy cows in Northern Ireland in 1992. Vet. Rec. 137:531-536.

Milian-Suazo, F., H. N. Erb, and R. D. Smith. 1988. Descriptive epidemiology of culling in dairy cows from 34 herds in New York State. Prev. Vet. Med. 6:243-251.

Nørgaard, N. H., K. M. Lind, and J. F. Agger. 1999. Cointegration analysis used in a study of dairy-cow mortality. Prev. Vet. Med. 42:99-119.

SAS Institute Inc. 2004. SAS OnlineDoc 9.1.2. http://support.sas. com/onlinedoc/912/docMainpage.jsp Accessed Oct. 28, 2007.

Sigurdarson, S., and T. Thorsteinsson. 1990. Sudden death of Icelandic dairy cattle. Vet. Rec. 127:410.

Smith, J. W., L. O. Ely, and A. M. Chapa. 2000. Effect of region, herd size, and milk production on reasons cows leave the herd. J. Dairy Sci. 83:2980-2987.
Stevenson, M. A., and I. J. Lean. 1998. Descriptive epidemiological study on culling and deaths in eight dairy herds. Aust. Vet. J. 76:482-488.

Stone, W. C., C. L. Guard, and D. A. Button. 2006. Why do cows die? Hoard's Dairyman 151:117.

Thomsen, P. T., and H. Houe. 2006. Dairy cow mortality. A review. Vet. Q. 28:122-129.

Thomsen, P. T., A. M. Kjeldsen, J. T. Sørensen, and H. Houe. 2004. Mortality (including euthanasia) among Danish dairy cows (1990-2001). Prev. Vet. Med. 62:19-33.

USDA. 2003. Transcript of Agriculture Secretary Ann M. Veneman announces additional protection measures to guard against BSE, Washington, DC, Dec. 30, 2003. Release no. 0450.03. Office of Communications, USDA, Washington, DC.

VanRaden, P. M., C. M. B. Dematawewa, R. E. Pearson, and M. E. Tooker. 2006. Productive life including all lactations and longer lactations with diminishing credits. J. Dairy Sci. 89:32133220 .

Weigel, K. A., R. W. Palmer, and D. Z. Caraviello. 2003. Investigation of factors affecting voluntary and involuntary culling in expanding dairy herds in Wisconsin using survival analysis. J. Dairy Sci. $86: 1482-1486$.

Young, A. 2002. Death loss of lactating cows-A major problem. http:// extension.usu.edu/files/publications/newsletter/pub_7133118. pdf Accessed Oct. 26, 2007. 